\title{
Evolving and intelligent systems applications special issue
}

\author{
Lazaros Iliadis $^{1} \cdot$ Costin Badica ${ }^{2}$
}

Published online: 7 January 2020

c) Springer-Verlag GmbH Germany, part of Springer Nature 2020

Intelligent Systems of the twenty-first century, have the ability to evolve over time in order to keep up with the dynamically changing global environment in every domain. Fuzzy, Neuro Fuzzy and Optimization Adaptive models often combining different algorithms in a Hybrid mode, constitute the core of Evolving Systems. This is the editorial of the "Evolving and Intelligent Systems Applications" Special Issue. It includes eight high quality scientific papers, presenting innovative research, falling in this area. They have been selected after passing successfully through a peer review process by independent academic referees.

The first paper "Fuzzy-statistical Prediction Intervals from Crisp Regression Models" is authored by Kingsley Adjenughwure and Basil Papadopoulos from the Democritus University of Thrace, Greece. It is a contribution with a certain level of novelty, related to the literature of fuzzy systems. It introduces an extended approach of constructing fuzzy numbers (representing uncertainties) from arbitrary statistical intervals. Moreover, two simple metrics are introduced capable of successfully evaluating the quality of the proposed fuzzy-statistical prediction intervals. The whole modeling approach is tested by applying two types of scenarios.

This is a paper authored by Kristiyan Balabanov, Frankfurt University of Applied Sciences Germany, Tymoteusz Cejrowsk, Gdansk University of Technology Poland, Doina Logofatu, Frankfurt University of Applied Sciences Germany and Costin Badica, University of Craiova Romania. The title of their paper is "Study on Population Dynamics for Triple-Linked Food Chain Using a Simulation-Based Approach". Simulation

Lazaros Iliadis

liliadis@civil.duth.gr

Costin Badica

cbadica@software.ucv.ro

1 School of Engineering, Democritus University of Thrace, Xanthi, Greece

2 Department of Computer and Information Technology, University of Craiova, Craiova, Romania has become a feasible testing approach that does not require investing valuable resources to create a concrete prototype, especially with the increasing computational power of computers. Thus, design changes can be adopted and design errors can be fixed before it is too late. Simulations have proven to be a cheap, safe and often more acceptable from an ethical perspective. This research paper discusses the results obtained by the analysis of a computational simulation of an elementary, yet analytically intractable problem scenario from the field of ecology. The actual goal achieved by this effort is the proof of concept that even with a seemingly simple agent-based model and simulation, it is possible to obtain plausible results regarding a system's real life behavior.

Omiros Iatrellis and Panos Fitsilis from the University of Thessaly Greece and Achilles Kameas from Hellenic Open University Greece, are the authors of the third paper entitled "EDUC8 pathways: Executing Self-evolving and Personalized Intra-organizational Educational Processes". This paper presents the EDUC8 platform that performs dynamic-evolving recommendation and execution of personalized education processes. The implemented prototype, aggregates a process execution engine, a rule engine and a semantic infrastructure for reconfiguring the learning pathways for each student. The semantic infrastructure consists of an ontology, enclosing the required knowledge and a semantic rule-set. During the execution of learning pathways, the system reasons over the rules and reconfigures the next steps of the learning process. The evolving knowledge base of the proposed platform comprises of new knowledge and facts which are developed based on the rule-base and the learning pathway meta-models established during execution.

An interesting research on the "Optimization of future charging infrastructure for commercial electric vehicles using a multi-objective genetic algorithm and real travel data" is authored by Li Zeng Volkswagen AG Hannover Germany, Timo Krallmann Hochschule Hannover Germany, Andrea Fiege, Marek Stess, Timo Graen and Michael Nolting, Volkswagen AG Hannover Germany. The automotive industry is gradually turning to the production of electric vehicle (EVs) for commercial customers. This contribution 
aims to develop a model that can successfully optimize the extension of public electrical charging facilities. The suitability of a massive conversion from combustion engines to EVs is evaluated for different scenarios. A multi-objective model using Genetic algorithms has been developed to evaluate the impact of an expanded charging infrastructure. The location and type of charging stations is optimized with respect to the number of failed trips due to empty batteries, and the overall infrastructure cost. Considering the fact that the use of commercial EVs will be unaltered after switching, travel data from vehicles with combustion engines have served as a starting point for the optimization of the charging infrastructure.

The fifth paper is entitled "A Hybrid Probabilistic Bi-Sector Fuzzy Regression Based Methodology for Normal Distributed Hydrological Variable" by Michail Spiliotis, Panagiotis Angelidis and Basil Papadopoulos, of the Democritus University of Thrace, Greece. The research described in this paper, introduces the use of the Tanaka fuzzy linear regression (TFLR) for the case of annual cumulative precipitation, by including all available historical data in the obtained fuzzy band. Moreover, a fuzzy bi-sector regression (BSR) is developed to address the determination of the return period for a given hydrological value. The BSR proposed herein, employs the inclusion property regarding the produced fuzzy band, similarly to the Tanaka fuzzy regression approach. This modeling algorithm performs fuzzy assessment of both the mean value and the standard deviation by following the TFLR process. Finally many indices are introduced that successfully validate the suitability of the proposed approach.

Argyro Mavrogiorgou, Athanasios Kiourtis and Dimosthenis Kyriazis from University of Piraeus, Greece are presenting a timely and interesting research in the sixth paper, The title is “A Plug'n'Play Approach for Dynamic Data Acquisition from Heterogeneous IoT Medical Devices of Unknown Nature". Actually, an evolving four step generic plug'n'play approach for healthcare Cyber-Physical systems is proposed and applied. Its contribution is the connection, recognition and integration of heterogeneous Internet of Things (IoT) devices of both known and unknown nature, so that they are capable of gathering data. Additionally they are simultaneously able to sense, monitor and interpret the different occasions under the environments in which they are used. The proposed mechanism is evaluated through a specific case, producing reliable results, thus being considered as a reference value of quality.

An evolving system entitled "Recommender Systems for IoT Enabled Quantified-Self Applications" is described in the seventh paper. The authors are: Seda Polat Erdeniz, Graz University of Technology Austria, Andreas Menychtas Bioassist, Athens Greece, Ilias Maglogiannis University of Piraeus Greece, Alexander Felfernig Graz University of Technology Austria and Thi Ngoc Trang Tran Graz University of
Technology, Austria. The research presented in this paper, introduces the following three novel Recommendation approaches for Quantified-Self Applications (QSA): Virtual Coach, Virtual Nurse, and Virtual Sleep Regulator. These approaches are helping QSA users to improve their health conditions. In fact, Virtual Coach operates like a real fitness coach to recommend personalized work-out plans, whereas Virtual Nurse considers the medical history and health targets of a user to recommend a suitable physical activity plan. Virtual Sleep Regulator is specifically designed for insomnia (a kind of sleep disorder) patients to improve their sleep quality with the help of recommended physical activity and sleep plans. A significant guidance on the optimal application of the recommender technologies is offered, based on the collected QS data aiming to develop qualitative recommendations for users' needs. The whole approach is tested based on a specific case of Virtual Sleep Regulator recommendation.

The eighth and last paper is entitled "Employing Query Disambiguation Using Clustering Techniques" and it is authored by Andreas Kanavos, Panagiota Kotoula, Christos Makris University of Patras Greece and Lazaros Iliadis, Democritus University of Thrace Greece. Due to the boundless expansion of the Web in the last decade, the research community has paid significant attention to the problem of effective searching through a vast volume of information. In this paper, the authors introduce a novel dynamic framework for improving information retrieval results. Initially, relevant documents are organized in clusters utilizing several metrics combined with language modelling tools. Then, a produced ranked list of the documents is returned to the users who have made a specific query, thus the scores assigned to the clusters and the query representations are extracted. This novel approach is based on the exploitation of the inter-documents similarities (lexical and/or semantics) which is performed after a sophisticated preprocessing step. The testing process has revealed that this algorithm can significantly improve the quality of the retrieved results.

We wish to express our appreciation and gratitude to the Editors in Chief of the Springer Evolving Systems Journal Professors P. Angelov, D. Filev, N. K. Kasabov, for their full support and for offering us their high quality journal for this Special Issue.

We have tried to make a minor contribution to the existing literature. We hope that the timely approaches presented in this publication will be appreciated by the international scientific community. We are certain that they will inspire further research on Intelligent Evolving applications in several and diverse domains.

The Guest Editors

Professor Lazaros S. Iliadis, Professor Costin Badica 